\title{
RANDOM SPARSE UNARY PREDICATES
}

\section{Introduction.}

Let $n$ be a positive integer, $0 \leq p \leq 1$. The random unary predicate $U_{n, p}$ is a probability space over predicates $U$ on $[n]=\{1, \ldots, n\}$ with the probabilities determined by

$$
\operatorname{Pr}[U(x)]=p, 1 \leq x \leq n
$$

and the events $U(x)$ being mutually independent over $1 \leq x \leq n$. Informally, we think of flipping a coin for each $x$ to determine if $U(x)$ holds, the coin coming up "heads" with probability $p$. We shall examine the first order language $\langle[n], \leq, U>$ with equality, a unary predicate $U$ and a binary predicate $\leq$. Examples of sentences in this language are:

$$
\begin{gathered}
A: \exists_{x} U(x) \\
B: \exists_{x} U(x) \wedge \forall_{y} \neg y<x \\
C: \exists_{x, y} U(x) \wedge U(y) \wedge \forall_{z} \neg[x<z \wedge z<y]
\end{gathered}
$$

$(>, \geq,<$ are natuarally definable from $\leq$ and equality.) For any such sentence $S$ we have the probability

$$
\operatorname{Pr}\left[U_{n, p}=S\right]
$$

While the use of unary predicates is natural for logicians there are two other equivalent formulations that will prove useful. We may think of $U$ as a subset of $[n]$ and speak about $i \in U$ rather than $U(i)$. Second we may associate with $U$ a sequence of zeroes and ones where the $i$-th term is one if $U(i)$ and zero if $\neg U(i)$. Thus we may talk of starting at $i$ and going to the next one. We shall use all three formulations interchangably.

Ehrenfeucht [??] showed that for any constant $p$ and any sentence $S$ in this language

$$
\lim _{n \rightarrow \infty} \operatorname{Pr}\left[U_{n, p} \models S\right]
$$

exists. In the case of sentences $A$ and $C$ the limiting probability is one. But sentence $B$ effectively states $1 \in U$, hence its limiting probability is $p$. We get around these edge effects with a new language, consisting of equality, a unary predicate $U$, and a ternary predicate $C$. We consider $C$ as a built in predicate on $[n]$ with $C(x, y, z)$ holding if and only if either $x<y<z$ 
or $y<z<x$ or $z<x<y$. Thinking of $[n]$ as a cycle, with 1 coming directly after $n, C(x, y, z)$ is the relation that $x$ to $y$ to $z$ goes in a clockwise direction. For any sentence $S$ in this new language we can again define $\operatorname{Pr}\left[U_{n, p} \models S\right]$ only in this case Ehrenfeucht's results give a Zero-One Law: for any constant $p$ and sentence $S$

$$
\lim _{n \rightarrow \infty} \operatorname{Pr}\left[U_{n, p} \models S\right]=0 \text { or } 1
$$

We shall call the first language the linear language and the second language the circular language. As a general guide, the circular language will tend to Zero-One Laws while the linear language, because of edge effects, will tend to limit laws.

We shall not restrict ourselves to $p$ constant but rather consider $p=p(n)$ as a function of $n$. We have in mind the "Evolution of Random Graphs" as first developed by Erdős and Rényi. Here as $p=p(n)$ evolves from zero to one the unary predicate evolves from holding for no $x$ to holding for all $x$. Analogously (but without formal definition) we have threshold functions for various properties. For example, $p(n)=n^{-1}$ is a threshold property for $A$. When $p(n) \ll n^{-1}$ almost surely $A$ fails while when $p(n) \gg n^{-1}$ almost surely $A$ holds. In Shelah,Spencer [??] we showed that when $p=n^{-\alpha}$ with $\alpha \in(0,1)$, irrational then a Zero-One Law held for the random graph $G(n, p)$ and in Euczak, Spencer [??] we found a near characterization of those $p=p(n)$ for which the Zero-One Law held. The situation with random unary predicates turns out to be somewhat simpler. Let us say $p=p(n)$ satisfies the Zero-One Law for circular unary predicates if for every sentence $S$ in the circular language

$$
\lim _{n \rightarrow \infty} \operatorname{Pr}\left[U_{n, p(n)} \models S\right]=0 \text { or } 1
$$

Here is our main result.

Theorem 1. Let $p=p(n)$ be such that $p(n) \in[0,1]$ for all $n$ and either

$$
p(n) \ll n^{-1}
$$

or for some positive integer $k$

$$
n^{-\frac{1}{k}} \ll p(n) \ll n^{-\frac{1}{k+1}}
$$

or for all $\epsilon>0$

$$
n^{-\epsilon} \ll p(n) \text { and } n^{-\epsilon} \ll 1-p(n)
$$


or for some positive integer $k$

$$
n^{-\frac{1}{k}} \ll 1-p(n) \ll n^{-\frac{1}{k+1}}
$$

or

$$
1-p(n) \ll n^{-1}
$$

Then $p(n)$ satisfies the Zero-One Law for circular unary predicates. Inversely if $p(n)$ falls into none of the above categories then it does not satisfy the Zero-One Law for circular unary predicates.

The inverse part is relatively simple. Let $A_{k}$ be the sentence that there exist $k$ consecutive elements $x_{1}, \ldots, x_{k} \in U$. $\quad(x, y$ are consecutive if for no $z$ is $C(x, z, y)$. For $k=2$ this is example C. ) Then $\operatorname{Pr}\left[A_{k}\right]$ is (for a given $n$ ) a monotone function of $p$. When $p(n) \sim c n^{-1 / k}$ and $c$ a positive constant the probability $\operatorname{Pr}\left[A_{k}\right]$ approaches a limit strictly between zero and one. (Roughly speaking, $n^{-1 / k}$ is a threshold function for $A_{k}$.) Thus for $p(n)$ to satisfy the Zero-One law we must have $p(n) \ll n^{-1 / k}$ or $p(n) \gg$ $n^{-1 / k}$. Further (replacing $U$ with $\neg U$ ), the same holds with $p(n)$ replaced by $1-p(n)$. For $p(n)$ to fall between these cracks it must be in one of the above five categories.

Remark. Dolan [??] has shown that $p(n)$ satisfies the Zero-One Law for linear unary predicates if and only if $p(n) \ll n^{-1}$ or $n^{-1} \ll p(n) \ll n^{-1 / 2}$ or $1-p(n) \ll n^{-1}$ or $n^{-1} \ll 1-p(n) \ll n^{-1 / 2}$. For $n^{-1 / 2} \ll p(n)=o(1)$ he considered the following property:

$D: \exists_{x} U(x) \wedge[U(x+1) \vee U(x+2)] \wedge \neg \exists_{y}[U(y) \wedge[U(y+1) \vee U(y+2)] \wedge y<x] \wedge U(x+1)$

(Addition is not in our language but we write $x+1$ as shorthand for that $z$ for which $x<z$ but there is no $w$ with $x<w<z$.) In our zero-one formulation $D$ basically states that the first time we have 11 comes before the first time we have 101. This actually has limiting probability .5. This example illustrates that limiting probability for linear unary predicates can depend on edge effects and not just edge effects looking at $U$ on a fixed size set $1, \ldots, k$ or $n, n-1, \ldots, n-k$. We defer our results for linear unary predicates to section 4 .

When $p(n) \ll n^{-1}$ the Zero-One Law is trivially satisfies since almost surely there is no $x$ for which $U(x)$. Also, if $p(n)$ satisfies the Zero-One Law 
so does $1-p(n)$. Suppose $p=p(n)$ satisfies $p(n) \gg n^{-\epsilon}$ and $1-p(n) \gg n^{-\epsilon}$ for all $\epsilon>0$. We show in a section 3 that for every $t$ there is a sequence $A_{1} \cdots A_{R}$ with the property that for any sentence $A$ of quantifier depth $t$ either all models $<[u], C, U>$ that contain $A_{1} \cdots A_{R}$ as a subsequence satisfy $A$ or no such models satisfy $A .\left(<[u], C, U>\right.$ contains $A_{1} \cdots A_{R}$ as a subsequence if for some $1 \leq j \leq u$ for all $1 \leq i \leq R$ we have $U(j+i)$ if and only if $x_{i}=1$, with $j+i$ defined modulo $u$.) For $p(n)$ in this range $<[u], C, U>$ almost surely contains any such fixed sequence $A_{1} \cdots A_{R}$ as a subsequence and hence the Zero-One Law is satisfied. This leaves us with only one case in Theorem 1, and that will be the object of the next section.

\section{The Main Case.}

Here we let $k$ be a positive integer and assume

$$
n^{-\frac{1}{k}} \ll p(n) \ll n^{-\frac{1}{k+1}}
$$

Our object is to show that $p(n)$ satisfies the Zero-One Law for circular unary predicates. We shall let $t$ be a fixed, though arbitrary large, positive integer. We shall examine the equivalence class under the $t$-move Ehrenfeucht game of the circular model. For the most part, however, we shall examine linear models.

We define (as Ehrenfeucht did) an equivalence class on models $M=<$ $n, \leq, U>$, two models $M, M^{\prime}$ being equivalent if they satisfy the same depth $t$ sentences or, equivalently, if the $t$-move Ehrenfeucht game on $M, M^{\prime}$ is won by the "Duplicator". The addition of models (with $M$ on $[n], M^{\prime}$ on $\left[n^{\prime}\right]$ we define $M+M^{\prime}$ on $\left[n+n^{\prime}\right]$ ) yields an addition of equivalence classes. We shall denote the equivalence classes by $x, y, \ldots$ and the sum by $x+y$. Results from the beautiful theory of these classes are given in Section 3.

Let us consider a random unary function $U$ defined on all positive integers $1,2, \ldots$ and with $\operatorname{Pr}[U(i)]=p$ for all $i$, these events mutually independent. (In the end only the values of $U(i)$ for $1 \leq i \leq n$ will "count" but allowing $U$ to be defined over all positive integers allows for a "fictitious play" that shall simplify the analysis.) Now for any starting point $i$ examine $i, i+1, \ldots$ until reaching the first $j$ (perhaps $i$ itself) for which $U(j)$. Call $[i, j]$ the 1-interval of $i$. (With probability one there will be such a $j$; fictitious play allows us to postpone the analysis of those negligible cases when no $j$ is found before $j>n$.) What are the possible Ehrenfeucht values of $<[i, j], \leq, U>$ ? The model must have a series of zeroes (i.e., $\neg U$ ) followed 
by one one (i.e., $U)$. There is an $s\left(s=3^{t}\right.$ will do) so that all such models with at least $s$ zeroes have the same Ehrenfeuct value. We can write these values as $a_{1}, \ldots a_{s}$ and $b$ ( $a_{i}$ having $i-1$ zeroes, $b$ having $s$ zeroes). Call this value the 1 -value of $i$. The probability of the 1-value being any particular $a_{i}$ is $\sim p$ while the probability of it being $b$ is $\sim 1$. (All asymptotics are as $p \rightarrow 0$.) We let $E_{1}$ denote this set of possible 1-values and we split $E_{1}=P_{1} \cup T_{1}$ with $P_{1}=\{b\}$ and $T_{1}=\left\{a_{1}, \ldots, a_{s}\right\}$. The 1 -values in $T_{1}$ we call 1-transient, the 1-value in $P_{1}$ we call 1-persistent.

Now (with an eye toward induction) we define the 2-interval of $i=i_{0}$. Take the 1 -interval of $i$, say $\left[i_{0}, i_{1}\right)$. Then take the 1 -interval of $i_{1}$, say $\left[i_{1}, i_{2}\right)$. Continue until reaching a 1 -interval $\left[i_{u}, i_{u+1}\right)$ whose 1 -value is 1 -transient. (Of course, this could happen with the very first interval.) We call $\left[i, i_{u+1}\right.$ ) the 2-interval of $i$. Now we describe the possible 2-values for this 2-interval. In terms of Ehrenfeucht value we can write the interval as $b+b+\ldots+b+a_{i}$ where there are $u$ (possibly zero) $b$ 's. Any $b+\ldots+b$ with at least $s$ addends $b$ has (see $\S 3.4$ ) the same value, call it $B$. Let $j b$ denote the sum of $j b$ 's. We define the transient 2 -values $T_{2}$ as those of the form $j b+a_{i}$ with $0 \leq j<s$ and the persistent 2-values $P_{2}$ as those of the form $B+a_{i}$. For example, let $t=5$ and $s=3^{5}=243$. Then $i$ has 2-value $6 b+a_{22}$ if, starting at $i$, six times there are at least 243 zeroes before a one and after the sixth one there are 21 zeroes and then a one. The 2 -value is $B+a_{5}$ if at least 243 times there are at least 243 zeroes before the next one and the first time two ones appear less than 243 apart they are exactly 5 apart. What are the probabilities for $i=i_{0}$ having any particular 2-value? The first 1-interval $\left[i_{0}, i_{1}\right)$ has distribution for 1 -value as previously discussed: $\sim p$ for each $a_{i}$ and $\sim 1$ for $b$. Having determined the first 1-interval the values starting at $i_{1}$ have not yet been examined. Hence the 1-value of the second 1-interval will be independent of the 1-value of the first and, in general, the sequence of 1 -values will be of mutually independent values. Then the transient 2values $j b+a_{i}$ each have probability $\sim p$ while the persistent 2 -values $B+a_{i}$ will each have probability $\frac{1}{s}+o(1)$. We let $P_{2}$ denote the set of persistent 2-values, $T_{2}$ the set of transient 2-values and $E_{2}=P_{2} \cup T_{2}$ the set of 2-values.

The 3 -value will contain all the notions of the general case. Beginning at $i=i_{0}$ take its 2 -interval $\left[i_{0}, i_{1}\right)$. Then take successive 2 -intervals $\left[i_{1}, i_{2}\right), \ldots,\left[i_{u-1}, i_{u}\right)$ until reaching an interval $\left[i_{u}, i_{u+1}\right)$ whose 2 -value is transient. The 3 -interval for $i$ is then $\left[i, i_{u+1}\right)$. Let $x_{1}, \ldots, x_{u}, y_{u+1}$ be the 2 -values for the successive intervals. Fromthe procedure all $x_{i} \in P_{2}$ while $y_{u+1} \in T_{2}$. Now consider (see $\S 3.1$ )the Ehrenfeucht equivalence classes (again with respect to a $t$-move game) over $\Sigma P_{2} .(\Sigma A$ is the set of strings 
over alphabet $A$.) Let $\alpha$ be the equivalence class for the string $x_{1} \cdots x_{u}$, then the 3 -value of $i$ is defined as the pair $\beta=\alpha y_{u+1}$. We let $E_{3}$ be the set of all such pairs and we call $\beta$ persistent (and place it in $P_{3}$ ) if $\alpha$ is a persistent state (as defined in $\S 3.2$ )in $\Sigma P_{2}$; otherwise we call $\beta$ transient and place it in $T_{3}$. If $x_{1} \cdots x_{u}$ and $x_{1}^{\prime} \cdots x_{u^{\prime}}^{\prime}$ are equivalent as strings in $P_{2}$ then $x_{1}+\ldots+x_{u}$ and $x_{1}^{\prime}+\ldots+x_{u^{\prime}}^{\prime}$ have (as shown in $\S 3.4$ ) the same Ehrenfeucht value. So the 3 -value of $i$ determines the Ehrenfeucht value of the 3-interval of $i$ though possibly it has more information. What are the probabilities for the 3 -value of $i$ ? Again we get a string of 2 -values $z_{1} z_{2} \ldots$ whose values are mutually independent and we stop when we hit a transient 2-value. We shall see (in the course of the full induction argument) that the probability of having 3-value $\beta$ is $\sim c_{\beta}$ for persistent $\beta$ and $\sim c_{\beta} p$ for transient $\beta$.

Now let us define $k$-interval and $k$-value, including the split into persistent and transient $k$-values by induction on $k$. Suppose $E_{k}, P_{k}, T_{k}$ have been defined. Beginning at $i=i_{0}$ let $\left[i_{0}, i_{1}\right)$ be the $k$-interval and then take succesive $k$-intervals $\left[i_{1}, i_{2}\right), \ldots,\left[i_{u-1}, i_{u}\right)$ until reaching a $k$-interval $\left[i_{u}, i_{u+1}\right)$ with transient $k$-value. Then $\left[i, i_{u+1}\right)$ is the $k+1$-interval of $i$. (Incidentally, suppose $U(i)$. Then $[i, i+1)$ is the 1 -interval of $i$ which is transient. But then $[i, i+1)$ is the 2 -interval of $i$ and is transient. For all $k[i, i+1)$ is the $k$-interval of $i$ and is transient.) Let $x_{1}, \ldots, x_{u}, y_{u+1}$ be the succesive $k$-values of the intervals. Let $\alpha$ be the equivalence class of $x_{1} \cdots x_{u}$ in $\Sigma P_{k}$. Then $i$ has $k+1$-value $\beta=\alpha y_{u+1}$. This value is persistent if $\alpha$ is persistent and transient if $\alpha$ is transient. This defines $E_{k+1}, P_{k+1}, T_{k+1}$, completing the induction. Our construction has assured that the $k$-value of $i$ determines the Ehrenfeucht value of the $k$-interval of $i$, though it may have even more information.

Now let us fix $i$ and look at the distribution of its $k$-value $V^{k}$. We show, by induction on $k$, that for every persistent $\beta \operatorname{Pr}\left[V^{k}=\beta\right]=c_{\beta}+o(1)$ while for every transient $\beta \operatorname{Pr}\left[V^{k}=\beta\right]=\left(c_{\beta}+o(1)\right) p$. Here each $c_{\beta}$ is a positive constant. Assume the result for $k$ and set $p_{\beta}=\operatorname{Pr}\left[V^{k}=\beta\right]$ for all $\beta \in E_{k}$. Let $p^{*}$ be the probability that $V^{k}$ is transient so that $p^{*} \sim c p, c$ a positive constant. Let $x_{1}, \ldots, x_{u}, y_{u+1}$ be the successive $k$ values of the $k$-intervals beginning at $i$, stopping at the first transient value. We can assume these values are taken independently from the inductively defined distribution on $E_{k}$. The distribution of the first transient value is the conditional distribution of $V^{k}$ given that $V^{k}$ is transient so the probability that it is some transient $y$ is $d_{y}+o(1)$ where $d_{y}=c_{y} / \sum c_{y^{\prime}}$, the sum over all transient $y^{\prime}$. Note all $d_{y}$ are positive constants.

The key to the argument is the distribution for the Ehrenfeucht equiva- 
lence class $\alpha$ for the finite sequence $x_{1} \cdots x_{u} \in \Sigma P_{k}$. Let $M$ be the set of all such equivalence classes. Let $L_{u}$ be the event that precisely $u$ persistent $x$ 's are found and then a transient $y$. Then $\operatorname{Pr}\left[L_{u}\right]=\left(1-p^{*}\right)^{u} p^{*}$ precisely. For $\beta \in P_{k}$ let $p_{\beta}^{+}=p_{\beta} /\left(1-p^{*}\right)$, the conditional probability that $V^{k}=\beta$ given that $V^{k}$ is persistent. Note that (as $p \rightarrow 0$ )

$$
p_{\beta}^{+} \sim p_{\beta} \sim c_{\beta}
$$

Conditioning on $L_{u}$ the $x_{1}, \ldots, x_{u}$ are mutually independent with distributions given by the $p_{\beta}^{+}$. Define on $M$ a Markov Chain (see $\S 3.3$ ) with transition probability $p_{\beta}^{+}$from and $\alpha$ to $\alpha+\beta$. We let $M(p)$ denote this Markov Chain. Observe that the set of states $M$ is independent of $p$ and the nonzeroness of the transition probabilities is independent of $p \in(0,1)$ though the actual transition probabilities do depend on $p$. There is a particular state $O$ representing the null sequence. Let $f(u, \alpha)$ be the probability of being at state $\alpha$ at time $u$, beginning at $O$ at time zero. Then $f(u, \alpha)$ is precisely the conditional distribution for $\alpha$ given $L_{u}$. But therefore, letting $W$ denote the Ehrenfeucht equivalence class,

$$
\operatorname{Pr}[W=\alpha]=\sum_{u=0}^{\infty} f(u, \alpha)\left(1-p^{*}\right)^{u} p^{*}
$$

Let $M^{o}$ be the Markov Chain on the same set with transition probability $c_{\beta}$ from $\alpha$ to $\alpha+\beta$ and let $f^{o}(u, \alpha)$ be the probability of going from $O$ to $\alpha$ in $u$ steps under $M^{o}$. Observe that $M^{o}$ is the limit of $M(p)$ as $p \rightarrow 0$ in that taking the limit of any (1-step) transition probability in $M(p)$ as $p \rightarrow \infty$ gives the transition probability in $M^{o}$.

Now we need some Markov Chain asymptotics. Assume $\alpha$ is transient. We claim (recall $\left.p^{*} \sim c p\right)$

$$
\operatorname{Pr}[W=\alpha] \sim\left[c \sum_{u=0}^{\infty} f^{o}(u, \alpha)\right] p
$$

and that the interior sum converges. In general the probability of remaining in a transient state drops exponentially in $u$ so there exist constants $K, \epsilon$ so that $f^{o}(u, \alpha)<K(1-\epsilon)^{u}$ for all $u$ giving the convergence. Moreover there exists $\epsilon_{1}, \epsilon_{2}, K_{1}$ so that for all $0<p<\epsilon_{1}$ we bound uniformly $f(u, \alpha)<$ $K_{1}\left(1-\epsilon_{2}\right)^{u}$ for all $u$. Pick $\epsilon_{3} \leq \epsilon_{1}$ so that for $0<p<\epsilon_{3}$ we have $p^{*} \leq 2 c p$. For any positive $\delta$ we find $U$ so that for $0<p<\epsilon_{3}$

$$
\frac{\sum_{u=U}^{\infty} f(u, \alpha)\left(1-p^{*}\right)^{u} p^{*}}{p}<\sum_{u=U}^{\infty} K_{1}\left(1-\epsilon_{2}\right)^{u} \frac{p^{*}}{p} \leq \frac{2 c K_{1}}{\epsilon_{2}}\left(1-\epsilon_{2}\right)^{U}<\frac{\delta}{2}
$$


For any fixed $0 \leq u<U$ we have $\lim _{p \rightarrow 0} f(u, \alpha)=f^{o}(u, \alpha)$ so that

$$
\lim _{p \rightarrow 0} \frac{\sum_{0 \leq u<U} f(u, \alpha)\left(1-p^{*}\right)^{u} p^{*}}{p}=\sum_{0 \leq u<U} c f^{o}(u, \alpha)
$$

With $U$ sufficiently large this may be made within $\delta / 2$ of $c \sum_{0}^{\infty} f^{o}(u, \alpha)$. But this holds for $\delta$ arbitrarily small, giving the claimed asymptotics of $\operatorname{Pr}[W=\alpha]$.

Remark. The rough notion here is that the probability of having a transient $k+1$-value is dominated by having few persistent $k$-intervals and then a transient $k$-interval. The transient 2 -intervals all had at most $s$ persistent 1-intervals. The situation changes with 3 -intervals. Recall $B a_{i}$ consisted of at least $s$ ones each preceeded by at least $s$ zeroes and then two ones $i$ apart. Consider an arbitrarily long grouping of 2-intervals of 2-value $B a_{i}$ but, say, with none of the form $B a_{3}$, i.e., 1001 not appearing and then, say, follow the last one, say $B a_{1}$, with a one so that the 3 - interval ends 111 . For every $u$ there is a $\sim c_{u} p$ probability of this being the 3 -interval with $u$ such 2-intervals and $c_{u}>0$ but all such 3 -intervals would be considered transient since a persistent sequence in $\Sigma P_{2}$ must surely contain every value in $P_{2}$.

Now suppose $\alpha$ is persistent. Again we have the precise formula

$$
\operatorname{Pr}[W=\alpha]=\sum_{u=0}^{\infty} f(u, \alpha)\left(1-p^{*}\right)^{u} p^{*}
$$

only this time it is the tail of the sum that dominates. As $\alpha$ is persistent there is a limiting probability $L=\lim _{u \rightarrow \infty} f^{o}(u, \alpha)$ with $L>0$ and furthermore the $M(p)$ approach $M^{o}$ in the sense that

$$
L=\lim _{p \rightarrow 0} \lim _{u \rightarrow \infty} f(u, \alpha)
$$

We claim

$$
\operatorname{Pr}[W=\alpha]=L+o(1)
$$

For any $\delta>0$ there exist $\epsilon$ and $U$ so that for $p \leq \epsilon$ and $u \geq U$ we have

$$
L-\delta<f(u, \alpha)<L+\delta
$$


Then, as $\sum_{u=0}^{\infty} L\left(1-p^{*}\right)^{u} p^{*}=L$,

$$
|\operatorname{Pr}[W=\alpha]-L| \leq \delta \sum_{u=U}^{\infty}\left(1-p^{*}\right)^{u} p^{*}+(L+1) \sum_{0 \leq u<U}\left(1-p^{*}\right)^{u} p^{*}
$$

For fixed $U$ the second sum is $o(1)\left(\right.$ as $p^{*} \rightarrow 0$ ) while the first sum is less than $\delta$ so the entire expression is less than $2 \delta$ for $p$ sufficiently small. As $\delta$ was arbitrary this gives the claim.

Recall that the $k+1$-value of the full $k+1$-interval is a pair consisting of the Ehrenfeucht value $W$ just discussed and the $k$-value of the first transient type $y_{u+1}$. The transient type's value has a limiting distribution which is independent of $W$, for conditional on any $L_{u}$ the distribution on $y_{u+1}$ is the same. All possible $y \in T_{k}$ have a limiting probability $d_{y} \in(0,1)$. Hence the probability of a $k+1$-value being $\beta=\alpha y$ is simply the product of the probabilities and hence approaches a constant if $\alpha$, and hence $\beta$, is persistent and is $\sim c p$ if $\alpha$, and hence $\beta$ is transient. This completes the inductive argument for the limiting probabilities of the $k$-values of the $k$-intervals.

We now let $L=L^{k}$ be the length of the $k$-interval of $i$ and find bounds on the distribution of $L$. A simple induction shows that if the sequence $1 \cdots 1$ of $k$ ones appears after $i$ then the $k$-interval of $i$ ends with this sequence or possibly before. Thus we get the crude bound

$$
\operatorname{Pr}[L>k a]<\left(1-p^{k}\right)^{a}
$$

so that asymptotically

$$
\operatorname{Pr}\left[L>\alpha p^{-k}\right]<e^{-c \alpha}
$$

where $c$ is a positive constant. In fact, this gives the correct order of magnitude, $L$ is (speaking roughly) almost always on the order of $p^{-k}$. We claim that there are positive constants $\epsilon_{t}, c_{t}$ so that

$$
\operatorname{Pr}\left[L^{t}>\epsilon_{t} p^{-t}\right]>c_{t}
$$

The argument is by induction, for $t=1$ the random variable $L^{1}$ is simply the number of trials until a success which occurs with probability $p$ and the distribution is easily computable. Assume this true for $t$ and let (as previously shown) $e_{t} p$ be the asymptotic probability that a $t$-interval will be transient. Pick $f_{t}$ positive with $f_{t} e_{t}<.5$. With probability at least .5 , the first $\gamma=f_{t} p^{-1} t$-intervals after $i$ will be persistent. Conditioning 
on an interval being persistent is conditioning on an event that holds with probability $1-o(1)$ so that each of these $t$-intervals will have length at least $\epsilon_{t} p^{-t}$ with probability at least $c_{t}-o(1)$. As the lengths are independent with conditional probability at least .99 at least $c_{t} \gamma / 2$ of the intervals have length at least $\epsilon_{t} p^{-t}$. Thus with probability at least, say .4 the total length $L^{t+1}$ is at least $c_{t} \gamma \epsilon_{t} p^{-t} / 2$ which is $\epsilon_{t+1} p^{-(t+1)}$ for an appropriate constant $\epsilon_{t+1}$, completing the induction.

Up to now the relation between $p$ and $n$, the number of integers, has not appeared. Recall that $p \rightarrow 0$ and $n \rightarrow \infty$ so that $n p^{k} \rightarrow \infty$ but $n p^{k+1} \rightarrow 0$. Now begin at $i=i_{0}=1$ and generate the $k$-interval $\left[i_{0}, i_{1}\right)$. Then generate the $k$-interval $\left[i_{1}, i_{2}\right)$ beginning at $i_{1}$ and continue. (We do this with $k$ fixed. Even if one of the intervals is transient we simply continue with $k$ intervals. Again we imagine continuing forever through the integers.) Let $N$ be that maximal $u$ for which $i_{u}-1 \leq n$, so that we have split $[n]$ into $N k$-intervals plus some excess. As each sequence of $k$ ones definitely will end a $k$-interval $N$ is at least the number of disjoint subintervals of $k$ ones. Simple expectation and variance calculations show that $N>.99 n p^{k}$ almost surely. On the other side set, with foresight, $c=4 c_{k}^{-1} \epsilon_{k}^{-1}$. If $N<c n p^{k}$ then the sum of the lengths of the first $c n p^{k} k$-intervals would be less than $n$. But these lengths are independent identically distributed variables and each length is at least $\epsilon_{k} p^{-k}$ with probability at least $c_{k}$ so that almost surely at least $c_{k} c n p^{k} / 2$ of them would have length at least $\epsilon_{k} p^{-k}$ and thus their total length would be at least $\left(c c_{k} \epsilon_{k} / 2\right) n>n$. That is, almost surely

$$
C_{1} n p^{k}<N<C_{2} n p^{k}
$$

where $C_{1}, C_{2}$ are absolute constants.

Let $\beta_{1}, \ldots, \beta_{N}$ be the $k$-values of the $k$-intervals generated by this procedure. Now we make two claims about this procedure. We first claim that almost surely none of the $\beta_{i}$ are transient. Each $\beta_{i}$ has probability $\sim c p$ of being transient so the probability that some $\beta_{i}, 1 \leq i \leq C_{2} n p^{k}$ is transient is at most $\sim(c p) C_{2} n p^{k}=\Theta\left(n p^{k+1}\right)=o(1)$. And almost surely $N<C_{2} n p^{k}$, proving the claim.

Let $A_{1} \cdots A_{R}$ be any fixed sequence of elements of $P_{k}$. The second claim is that almost surely $A_{1} \cdots A_{r}$ appears as a subsequence of the $\beta$ sequence, more precisely that almost surely there exists $i$ with $1 \leq i \leq N-R$ so that $\beta_{i+j}=A_{j}$ for $1 \leq j \leq R$. (For technical reasons we want the subsequence not to start with $\beta_{1}$.) As each $\beta_{i}$ has a positive probability of being any particular $x \in P_{k}$ and the $\beta_{i}$ are independent and $C_{1} n p^{k} \rightarrow \infty$ almost surely 
this fixed sequence will appear in the first $C_{1} n p^{k} \beta^{\prime}$ s. And almost surely $N>C_{1} n p^{k}$, proving the claim.

We have a third claim that is somewhat technical. For any $1 \leq j \leq k$ let $\beta_{1}, \ldots, \beta_{u}$ denote the $j$-values of the successive $j$-intervals starting at one, where $\beta_{u}$ is the last such interval that is in $P_{j}$. We know that almost surely $\beta_{1} \cdots \beta_{u}$ is persistent in $\Sigma P_{j}$. We claim further that almost surely $\beta_{2} \beta_{3} \cdots \beta_{u}$ is persistent in $\Sigma P_{j}$. It suffices to show this for any particular $j$ as there are only a finite number of them. For any integer $A$ we have $u-1 \geq A$ almost surely and the probability that $\beta_{2} \cdots \beta_{A+1}$ is transient goes to zero with $A$ so almost surely $\beta_{2} \cdots \beta_{u}$ is persistent. Let us call $[b, c)$ a super $k$-interval (for a given $U$ ) if it is a $k$-interval and further for every $1 \leq j \leq k$ letting $\beta_{1}, \ldots, \beta_{u}$ be the successive $j$-values of the $j$-intervals beginning at $b$ and stopping with the last persistent value - that then $\beta_{2} \beta_{3} \cdots \beta_{u}$ is persistent in $\Sigma P_{j}$. So almost surely the $k$-interval $\left[1, i_{1}\right)$ is a super $k$-interval.

We shall show, for an appropriate sequence $A_{1}, \ldots, A_{R}$, that all $U$ satisfying the above three claims give models $\langle n, C, U\rangle$ which have the same Ehrenfeucht value.

We first need some glue. Call $[a, b)$ an incomplete $k$-interval (with respect to some fixed arbitrary $U$ ) if the $k$-interval beginning at $a$ is not completed by $b-1$. Suppose $[a, b)$ is an incomplete $k$-interval and $[b, c)$ is a persistent super $k$-interval. We claim $[a, c)$ is a persistent $k$-interval. The argument is by induction on $k$. For $k=1,[a, b)$ must consist of just zeroes while $[b, c)$ consists of at least $s$ zeroes followed by a one. But then so does $[a, c)$. Assume the result for $k$ and let $[a, b)$ be an incomplete $k+1$-interval and $[b, c)$ be a persistent $k+1$-interval. We split $[a, b)$ into a (possibly empty) sequence $x_{1}, x_{2}, \ldots, x_{r}$ of persistent $k$-intervals followed by (possibly null) incomplete $k$-interval $\left[a^{+}, b\right)$ with value, say, $y$. We split $[b, c)$ (renumbering for convenience) into a sequence $x_{r+1}, \ldots, x_{s}, y_{s+1}$ of $k$-intervals, all persistent except the last which is transient. Then, by induction, $y+x_{r+1}$ is a persistent $k$-interval with some value $x_{r+1}^{\prime}$. Then $[a, c)$ splits into $k$-intervals with values $x_{1}, \ldots, x_{r}, x_{r+1}^{\prime}, x_{r+2}, \ldots, x_{s}, y_{s+1}$. By the super-persistency $x_{r+2} \cdots x_{s}$ is persistent in $\Sigma P_{k}$ and hence (see $\S 3.2$ ) so is $x_{1} \cdots x_{r} x_{r+1}^{\prime} x_{r+2} \cdots x_{s}$ and therefore $[a, c)$ is a persistent $k+1$ - interval.

Now let $\langle[n], C, U>$ be any model that meets the three claims above, all of which hold almost surely for $p$ in this range. We set $i=i_{0}=1$ and find successive $k$-intervals $\left[i_{0}, i_{1}\right),\left[i_{1}, i_{2}\right), \ldots$ until $\left[i_{u-1}, i_{u}\right)$ and then $U$ on $\left[i_{u}, n\right]$ gives an incomplete $k$-interval. By the third claim $\left[1, i_{1}\right)$ is superpersistent and so the "interval" $\left[i_{u}, n\right] \cup\left[1, i_{1}\right)$ (going around the corner) is $k$-persistent. Hence we have split $[n]$ (now thinking of it as a cycle with 1 following $n$ ) 
into $k$-persistent intervals with $k$-values $x_{1}, x_{2}, \ldots, x_{u}$. The $k$-value for $x_{1}$ may be different from that for $\left[1, i_{1}\right)$ but the others have remained the same. This sequence contains the sequence $A_{1} \cdots A_{R}$ described in $\S 3.5$. But this implies (see §3.6) that the Ehrenfeuct value is determined, completing the proof.

\section{Background.}

\subsection{The Ehrenfeucht Game.}

Let $A$ be a fixed finite alphabet (in application $A$ is $P_{k}$ or $\{0,1\}$ ) and $t$ a fixed positive integer. We consider the space $\Sigma A$ of finite sequences $a_{1} \cdots a_{u}$ of elements of $A$. We can associate with each sequence a model $<[u], \leq, f>$ where $f:[u] \rightarrow A$ is given by $f(i)=a_{i}$. For completeness we describe the $t$-round Ehrenfeucht Games on sequences $a_{1} \cdots a_{u}$ and $a_{1}^{\prime} \cdots a_{u^{\prime}}^{\prime}$. There are two players, Spoiler and Duplicator. On each round the Spoiler first selects one term from either sequencs and then the Duplicator chooses a term from the other sequence. Let $i_{1}, \ldots i_{t}$ be the indices of the terms chosen from the first sequence, $i_{q}$ in the $q$-th round and let $i_{1}^{\prime}, \ldots i_{t}^{\prime}$ denote the corresponding indices in the second sequence. For Duplicator to win he must first assure that $a_{i_{q}}=a_{i_{q}^{\prime}}^{\prime}$ for each $q$, i.e. that he selects each round the same letter as Spoiler did. Second he must assure that for all $a, b$

$$
i_{a}<i_{b} \Leftrightarrow i_{a}^{\prime}<i_{b}^{\prime} \text { and } i_{a}=i_{b} \Leftrightarrow i_{a}^{\prime}=i_{b}^{\prime}
$$

(It is a foolish strategy for Spoiler to pick an already selected term since Duplicator will simply pick its already selected counterpart but this possiblity comes in in the Recursion discussed later.) This is a perfect information game so some player will win. Two sequences are called equivalent if Duplicator wins. Ehrenfeucht showed that this is an equivalence class and that two sequences are equivalent if their models have the same truth value on all sentences of quantifier depth at most $t$. We let $M$ denote the set of equivalence classes which is known to be a finite set. $\Sigma A$ forms a semigroup under concatenation, denoted + , and this operation filters to an operation, also denoted + , on $M$. We use $x, y, \ldots$ to denote elements of $M: x+y$ their sum; $O$ is the equivalence class of the null sequence which acts as identity. We associate $a \in A$ with the sequence $a$ of length one and its equivalence class (which contains only it), also called $a$. We let $j x$ denote $x+\ldots+x$ with $j$ summands. From analysis of the Ehrenfeucht game (see §3.4) it is 
known that there exists $s$ (for definiteness we may take $s=3^{t}$ ) so that:

$$
j x=k x \text { for all } j, k \geq s, x \in M
$$

Example. With $A=\{0,1\}$ we naturally associate sequences such as 101 with $<\{1,2,3\}, \leq, f>$ with $f(1)=1, f(2)=0, f(3)=1$. The addition of 101 and 1101 is their concatenation (in that order) 1011101. The first order language has as atomic formulas $x \leq y, x=y$ and $f(x)=a$ for each $a \in A$. The sentence

$$
\exists_{x} \exists_{y} \exists_{z} f(x)=1 \wedge f(y)=0 \wedge f(z)=1 \wedge x<y \wedge y<z
$$

is satisfied by 01110001 but not by 000111000 so these are in different equivalence classes with $t=3$. We could also write that 101 appears as consecutive terms with

$\exists_{x} \exists_{y} \exists_{z} f(x)=1 \wedge f(y)=0 \wedge f(z)=1 \wedge x<y \wedge y<z \wedge \neg \exists_{w}[(x<w \wedge w<y) \vee(y<w \wedge w<z)]$

Informally we would just say $\exists_{x} f(x)=f(x+1)=f(x+2)=1$ but the quantifier depth is four.

\subsection{Persistent and Transient.}

Definition and Theorem. We call $x \in M$ persistent if

$$
\begin{gathered}
\forall_{y} \exists_{z} x+y+z=x \\
\forall_{y} \exists_{z} z+y+x=x \\
\exists_{p} \exists_{s} \forall_{y} p+y+s=x
\end{gathered}
$$

These three properties are equivalent. We call $x$ transient if it is not persistent.

Proof of Equivalence.

$(3) \Rightarrow(1):$ Take $z=s$, regardless of $y$. Then

$$
x+y+z=(p+y+s)+y+s=p+(y+s+y)+s=x
$$

$(1) \Rightarrow(3)$ : Let $R_{x}=\{x+v: v \in M\}$. We first claim there exists $u \in M$ with $\left|R_{x}+u\right|=1$, i.e., all $x+y+u$ the same. Otherwise take $u \in M$ with $\left|R_{x}+u\right|$ minimal and say $v, w \in R_{x}+u$. As $R_{x}+u \subseteq R_{x}$ we write 
$v=x+u_{1}, w=x+u_{2}$. From(1), with $y=u_{1}$, we have $x=v+u_{3}$ and thus $w=v+u_{4}$ with $u_{4}=u_{3}+u_{2}$. Then

$$
w+s u_{4}=v+(s+1) u_{4}=v+s u_{4}
$$

Adding $s u_{4}$ to $R+u$ sends $v, w$ to the same element so $\left|R+u+s u_{4}\right|<|R+u|$, contradicting the minimality. Now say $R_{x}+u=\left\{u_{5}\right\}$. Again by (1) there exists $u_{6}$ with $u_{5}+u_{6}=x$. Then $R_{x}+\left(u+u_{6}\right)=\{x\}$ so that (3) holds with $p=x, s=u+u_{6}$.

By reversing addition (noting that (3) is selfdual while the dual of (1) is (2)) these arguments give that (3) and (2) are equivalent, completing the proof.

Let $x$ be persistent and consider $v=x+y$. Let $z$ be such that $x+w+z=$ $x$ for all $w$. Then for all $w v+w+(z+y)=(x+(y+w)+z)+y=x+y=v$ and hence $v$ is persistent. Dually,if $x$ is persistent $y+x$ is persistent. Together

$$
\text { If } x \text { is persistent then } w_{1}+x+w_{2} \text { is persistent }
$$

for any $w_{1}, w_{2} \in M$.

From (1) the relation $x \equiv_{R} u$ defined by $\exists_{v}(x+v=u)$ is an equivalence relation on the set of persistent $x \in M$. We let $R_{x}$ denote the $\equiv_{R^{-}}$class containing $x$ so that

$$
R_{x}=\{x+v: v \in M\}
$$

From(2) the relation $x \equiv_{L} u$ defined by $\exists_{v}(v+x=u)$ is also an equivalence relation on the set of persistent $x \in M$. We let $L_{x}$ denote the $\equiv_{L^{-}}$-class containing $x$ so that $L_{x}=\{v+x: v \in M\}$. Let $x$ be persistent and let $p, s$ (by (3)) be such that $p+z+s=x$ for all $x$. Setting $z=O, x=p+s$. Thus for all $z$

$$
x+z+x=(p+s)+z+(p+s)=p+(s+z+p)+s=x
$$

Let $R_{x}, L_{y}$ be equivalence classes under $\equiv_{R}, \equiv_{L}$ respectively. Then $x+$ $y \in R_{x} \cap L_{y}$. Let $z \in R_{x} \cap L_{y}$. Then there exist $a, b$ with $x=z+a$ and $y=b+z$ so that $x+y=z+(a+b)+z$. But as $z$ is persistent the above argument (with $z$ as $x$ and $a+b$ as $z$ ) gives $z+(a+b)+z=z$. Thus

$$
R_{x} \cap L_{y}=\{x+y\} \text { for all persistent } x, y
$$

Remarks. Let $A=\{0.1\}$. A sequence $a_{1} \cdots a_{u}$ is transient if and only if there is a sentence $Q$ of quantifier depth at most $t$ so that $a_{1} \cdots a_{u}$ fails $Q$ 
but there is an extension to $a_{1} \cdots a_{u} a_{u+1} \cdots a_{v}$ which satisfies $Q$ such that all further extensions $a_{1} \cdots a_{v} a_{v+1} \cdots a_{w}$ also satisfy $Q$. For example, with $t=4$, let $Q$ be the existence of a block 101. If a sequence does not satisfy $Q$ then the extension given by adding 101 does satisfy $Q$ and all further extensions will satisfy $Q$. Thus for $a_{1} \cdots a_{u}$ to be persistent for $t=4$ it must contain 101 and indeed all blocks of length three. We think of property (3) of persistency as indicating that a persistent sequence is characterized by $p$, its prefix, and $s$, its suffix. There are properties such as $\exists_{x} f(x)=1 \wedge \neg \exists_{y} y<x$ that depend on the left side of the sequence, in this case the value $f(1)$. There are other properties such as $\exists_{x} f(x)=1 \wedge \neg \exists_{y} x<y$ which depend on the right side of the sequence. There will be sequences with values $p, s$ for the left and right side respectively so that the Ehrenfeucht value of the sequence is now determined, regardless of what is placed in the middle.

Remarks. Certain sentences $Q$ have the property that if any $a_{1} \cdots a_{u}$ satisfies $Q$ then all extensions $a_{1} \cdots a_{u} a_{u+1} \cdots a_{v}$ satisfy $Q$. The sentence that the first term of the sequence is 1 has this property; the sentence that the last term of the sequence is 1 does not have this property. Call such properties unrighteous, as they (roughly) do not depend on the right hand side of the sequence. Sequences with Ehrenfeucht value in a given $R_{x}$ all have the same truth value for all unrighteous properties. Sequences with Ehrenfeucht value in a given $L_{x}$ would all have the same truth value for all (correspondingly defined) unleftuous properties.

\subsection{The Markov Chain.}

Now consider a probability distribution over $A$, selecting each $a$ with nonzero probability $p_{a}$. This naturally induces a distribution over $A^{u}$, the sequences of length $u$, assuming each element is chosen independently. This then leads to a distribution over the equivalence classes $M$. For all $u \geq 0, x \in M$ let $P_{u}(x)$ be the probability that a random string $a_{1} \cdots a_{u}$ is in class $x$. On $M$ we define a Markov Chain, for each $x$ the transition probability from $x$ to $x+a$ being $p_{a}$.

In Markov Chain theory the states $x \in M$ are split into persistent and transient, a state $x$ is persistent if and only if it lies in a minimal closed set. We claim Markov Chain persistency is precisely persistency as defined by (1), (2), (3). If $C$ is closed and $x \in C$ then $R_{x} \subseteq C$ and $R_{x}$ is itself closed. If $x$ satisfies (1) then $R_{u}=R_{x}$ for all $u=x+y \in R_{x}$ so $x$ is Markov Chain 
persistent. Conversely if $x$ is Markov Chain persistent then $R_{x}$ must be minimal closed so $R_{u}=R_{x}$ for all $u=x+y \in R_{x}$ and so $x$ satisfies (1).

The Markov Chain $M$ restricted to a minimal closed set $R_{x}$ is aperiodic since $x+s a \in R_{x}$ and $(x+s a)+a=x+s a$. Hence from Markov Chain theory when $x$ is persistent $\lim _{u \rightarrow \infty} P_{u}(x)$ exists.

A random walk on $M$, beginning at $O$, will with probability one eventually reach a minimal closed set $R_{x}$ and then it must stay in $R_{x}$ forever. Let $P\left[R_{x}\right]$ denote the probability that $R_{x}$ is the closed state reached.

\subsection{Recursion.}

Again let $A$ be a finite alphabet, $M$ the set of equivalence classes of $\Sigma A$ and now specify some $B \subseteq M$. As $B$ is also a finite set we can define equivalence classes (with respect to the same constant $t$ ) on $\Sigma B$, let $M^{+}$denote the set of such classes. Now let $b_{1} \cdots b_{u}$ and $b_{1} \cdots b_{u^{\prime}}^{\prime}$ be equivalent sequences of $\Sigma B$. We claim that

$$
b_{1}+\ldots+b_{u}=b_{1}^{\prime}+\ldots+b_{u^{\prime}}^{\prime}
$$

as elements of $M$. Let $s_{1}, \ldots, s_{u}, s_{1}^{\prime}, \ldots, s_{u^{\prime}}^{\prime}$ be specific elements of $\Sigma A$ in the repective $b_{i}$ or $b_{i}^{\prime}$ classes. It suffices to give a strategy for Duplicator with models $s_{1}+\ldots+s_{u}$ and $s_{1}^{\prime}+\ldots+s_{u^{\prime}}^{\prime}$. Suppose Spoiler picks an element $x$ in, say, some $s_{i}$. In the game on $\Sigma B$ we know Duplicator has a winning reply to $b_{i}$ of some $b_{i^{\prime}}^{\prime}$. Now Duplicator will pick some $x^{\prime}$ in $s_{i^{\prime}}^{\prime}$. To decide the appropriate $x^{\prime}$ in $s_{i^{\prime}}^{\prime}$ to pick Duplicator considers a subgame on $s_{i}$ and $s_{i^{\prime}}^{\prime}$. As these are equivalent Duplicator will be able to find such $x^{\prime}$ for the at most $t$ times that he is required to.

This general recursion includes the previous statement that for all $j, k \geq$ $s$ and any $x \in M$ we have $j x=k x$. Here $B=\{x\}$ and this says that Duplicator can win the $t$-move Ehrenfeucht game between a sequence of $j x$ 's and a sequence of $k x$ 's; that is, that $<[j], \leq>$ and $<[k], \leq>$ are equivalent a basic result on Ehrenfeucht games. In our argument we apply it inductively with $A=P_{k}$. We know, inductively, that all $k$-intervals having the same $k$-value $x \in P_{k}$ have the same Ehrenfeucht value. Now the $k+1$-interval of $i$ is associated with a sequence $x_{1} \cdots x_{u} \in \Sigma P_{k}$ and a "tail" $y_{u+1} \in T_{k}$. We call two such $k+1$-intervals equivalent if $x_{1} \cdots x_{u^{\prime}}^{\prime}$ and $x_{1}^{\prime} \cdots x_{u^{\prime}}^{\prime}$ are equivalent in $\Sigma P_{k}$ and $y_{u+1}=y_{u^{\prime}+1}^{\prime}$. Now $x_{1}+\ldots+x_{u}=x_{1}^{\prime}+\ldots+x_{u^{\prime}}^{\prime}$ and so the $k+1$-intervals have equal Ehrenfeucht value. 


\subsection{Cycles.}

Again let $M$ be the set of equivalence classes on $\Sigma A$. Now consider cycles $a_{1} \cdots a_{u}$ (thinking of $a_{1}$ following $a_{u}$ ) with $a_{i} \in A$ and consider equivalence classes under the $(t+1)$-move Ehrenfeucht game. Here we must preserve the ternary clockwise predicate $C(x, y, z)$. Any first move $a_{i}$ reduces the cycle to a linear structure $a_{i} \cdots a_{u} a_{1} \cdots a_{i-1}$ of the form $\left.<[u], \leq, f\right\rangle$ with an Ehrenfeucht value $x=x_{i}$. Two cycles are equivalent if they yield the same set of values $x_{i} \in M$.

For every persistent $x \in M$ let (by (3)) $p=p_{x}, q=s_{x}$ be such that $x=p_{x}+y+s_{x}$ for all $y \in M$. Let $P_{x}$ and $S_{x}$ be fixed sequences (i.e., elements of $\Sigma A$ ) for these equivalence classes and let $R_{x}$ be the sequence consisting of $S_{x}$ in reverse order followed by $P_{x}$. If the cycle $a_{1} \cdots a_{u}$ contains $R_{x}$ as a subsequence then selecting $a_{i}$ as the first element of $P_{x}$ gives a linear structure beginning with $P_{x}$ and ending with $S_{x}$, hence of value $p_{x}+y+s_{x}=$ $x$.

Let $R \in \Sigma A$ be a specific sequence given by the concatenation of the above $R_{x}$ for all persistent $x \in M$. Then we claim $R$ is a universal sequence in the sense that all $a_{1} \cdots a_{u} \in \Sigma A$ (for any $u$ ) that contain $R$ as a subsequence are equivalent. For every persistent $x \in M$ there is an $a_{i}$ so that $a_{i} \cdots a_{i-1}$ has value $x$. Conversely every $a_{i}$ belongs to at most one of the $R_{x}$ creating $R$ (maybe none if $a_{i}$ isn't part of $R$ ) and so there will be an $R_{x}$ not containing that $a_{i}$. Then in $a_{i} \cdots a_{i-1}$ the subsequence $R_{x}$ will appear as an interval. Hence the value of $a_{i} \cdots a_{i-1}$ can be written $w_{1}+x+w_{2}$, which is persistent. That is, the values of $a_{i} \cdots a_{i-1}$ are precisely the persistent $x$ and hence the class of $a_{1} \cdots a_{u}$ in the circular $t+1$-Ehrenfeucht game is determined.

\subsection{Recursion on Cycles.}

Again let $A$ be a finite alphabet, $M$ the set of equivalence classes in $\Sigma A$ and specify some $B \subseteq M$. Suppose a cycle $a_{1} \cdots a_{u}$ on $A$ may be decomposed into intervals $s_{1}, \ldots, s_{r}$ with Ehrenfeucht values $b_{1} \cdots b_{r}$. Then the Ehrenfeucht value of the cycle $b_{1} \cdots b_{r}$ determines the Ehrenfeucht value of $a_{1} \cdots a_{u}$. The argument is the same as for recursion on intervals. Let $a_{1} \cdots a_{u}$ and $a_{1}^{\prime} \cdots a_{u^{\prime}}^{\prime}$ be decomposed into $s_{1} \cdots s_{r}$ and $s_{1}^{\prime} \cdots s_{r^{\prime}}^{\prime}$ with Ehrenfeucht values $b_{1} \cdots b_{r}$ and $b_{1} \cdots b_{r^{\prime}}^{\prime}$. Spoiler picks $x$ in some $s_{i}$. In the game on cycles over $B$ Duplicator can respond $b_{i^{\prime}}^{\prime}$ to $b_{i}$. Then Duplicator picks an $x^{\prime} \in s_{i^{\prime}}^{\prime}$ so that he can win the subgame on $s_{i}$ and $s_{i^{\prime}}^{\prime}$.

We apply this is $\S 2$ with $A=\{0,1\}$ and $B=P_{k}$. Here the $\beta \in P_{k}$ 
may have more information than the Ehrenfeucht value but this only helps Duplicator.

\section{The Linear Model.}

We have already remarked in $\S 1$ that Zero-One Laws generally do not hold for the linear model $\langle[n], \leq, U>$ and that P. Dolan has characterized those $p=p(n)$ for which they do. Our main object in this section is the following convergence result.

Theorem 2. Let $k$ be a positive integer, and $S$ a first order sentence. Then there is a constant $c=c_{k, S}$ so that for any $p=p(n)$ satisfying

$$
n^{-\frac{1}{k}} \ll p(n) \ll n^{-\frac{1}{k+1}}
$$

we have

$$
\lim _{n \rightarrow \infty} \operatorname{Pr}\left[U_{n, p} \models S\right]=c
$$

Again we shall fix the quantifier depth $t$ of $S$ and consider Ehrenfeucht classes with respect to that $t$. For each $\beta \in P_{k}$ let $c_{\beta}$ be the constant defined in $\S 2$ as the limiting probability that a $k$-interval has $k$-value $\beta$. Let $M$ be the set of equivalence classes of $\Sigma P_{k}$, a Markov Chain as defined in $\S 3$, and for each $\equiv_{R^{\text {-class }}} R_{x}$ let $P\left[R_{x}\right]$, as defined in $\S 3$, be the probability that a random sequence $\beta_{1} \beta_{2} \cdots$ eventually falls into $R_{x}$.

In $<[n], \leq, U>$ let $\beta_{1} \cdots \beta_{N}$ denote the sequence of $k$-values of the successive $k$-intervals, denoted $\left[1, i_{1}\right),\left[i_{1}, i_{2}\right), \ldots$, from 1 .

Set, with foresight, $\delta=10^{-2} 3^{-t}$.

We shall call $U$ on $[n]$ right nice if it satisfies two conditions. The first is simply that all the $\beta_{1}, \ldots, \beta_{N}$ described above are persistent. We know from $\S 2$ that this holds almost surely. The second will be a particular universality condition. Let $A_{1} \cdots A_{R}$ be a specific sequence in $\Sigma P_{k}$ with the property that for every $R_{x}$ and $L_{y}$ there exists a $q$ so that

$$
A_{1} \cdots A_{q} \in L_{y} \text { and } A_{q+1} \cdots A_{R} \in R_{x}
$$

(We can find such a sequence for a particular choice of $R_{x}$ and $L_{y}$ by taking specific sequences in $\Sigma P_{k}$ in those classes and concatenating them. The full sequence is achieved by concatenting these sequences for all choices of $R_{x}$ and $L_{y}$. Note that as some $A_{1} \cdots A_{q} \in L_{y}$ the full sequence is persistent.) The second condition is that inside any interval $[x, x+\delta n] \subset[1, n]$ there exist $R$ consecutive $k$-intervals $\left[i_{L}, i_{L+1}\right), \ldots,\left[i_{L+R}, i_{L+R+1}\right)$ whose $k$-values 
are, in order, precisely $A_{1}, \ldots, A_{R}$. We claim this condition holds almost surely. We can cover $[1, n]$ with a finite number of intervals $\left[y, y+\frac{\delta}{3} n\right]$ and it suffices to show that almost always all of them contain such a sequence, so it suffices to show that a fixed $\left[y, y+\frac{\delta}{3} n\right]$ has such a sequence. Generating the $k$-intervals from 1 almost surely a $k$-interval ends after $y$ and before $y+\frac{\delta}{6} n$. Now we generate a random sequence $\beta_{1} \cdots$ on an interval of length $\frac{\delta}{6} n$. But constants do not affect the analysis of $\S 2$ and almost surely $A_{1} \cdots A_{R}$ appears.

Now on $<[n], \leq, U>$ define $U^{r}$ by $U^{r}(i)$ if and only if $U(n+1-r)$. $U^{r}$ is the sequence $U$ in reverse order. Call $U$ left nice if $U^{r}$ is right nice. Call $U$ nice if it is right nice and left nice. As all four conditions hold almost surely, the random $U_{n, p}$ is almost surely nice.

Let $U$ be nice and let $\beta_{1} \cdots \beta_{N}$ and $\beta_{1}^{r} \cdots \beta_{N^{r}}^{r}$ denote the sequences of $k$ values for $U$ and $U^{r}$ respectively and let $R_{x}$ and $R_{x^{r}}$ denote their $\equiv_{R^{-c l a s s e s}}$ respectively. (Both exist since the sequences are persistent.)

Claim. The values $R_{x}$ and $R_{x^{r}}$ determine the Ehrenfeucht value of nice $U$.

We first show that Theorem 2 will follow from the Claim. Let $R_{x}, R_{x^{r}}$

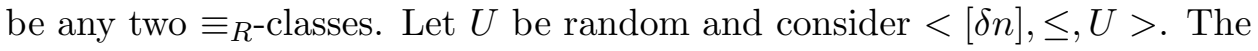
sequence of $k$-values lies in $R_{x}$ with probability $P\left[R_{x}\right]+o(1)$. The same holds for $U^{r}$ on $[\delta n]$. But $U^{r}$ examines $U$ on $[(1-\delta) n, n]$ so as $\delta<.5$ the values of the $\equiv_{R}$-classes are independent and so the joint probability of the values being $R_{x}$ and $R_{x^{r}}$ respectively is $P\left[R_{x}\right] P\left[R_{x^{r}}\right]+o(1)$. Given the Claim $<[n], \leq, U>$ would then have a value $v=v\left(R_{x}, R_{x^{r}}\right)$. As

$$
\sum P\left[R_{x}\right] P\left[R_{x^{r}}\right]=\sum P\left[R_{x}\right] \sum P\left[R_{x^{r}}\right]=1 \times 1=1
$$

this would give a limiting distribution for the Ehrenfeucht value $v$ on $<$ $[n], \leq, U>$.

Now for the claim. Fix two models $M=<[n], \leq, U>$ and $M^{\prime}=<\left[n^{\prime}\right], \leq$ , $U^{\prime}>$, both nice and both with the same values $R_{x}, R_{x^{r}}$. Consider the $t$ move Ehrenfeucht game. For the first move suppose Spoiler picks $m \in M$. By symmetry suppose $m \leq \frac{n}{2}$. Let $\left[i_{r-1}, i_{r}\right)$ be one of the $k$-intervals with, say, .51n $\leq i_{r} \leq .52 n$. We allow Duplicator a "free" move and have him select $i_{r}$. Let $\beta_{1} \cdots \beta_{N}$ and $\beta_{1}^{\prime} \cdots \beta_{N^{\prime}}^{\prime}$ be the sequences of $k$-values for $M$ and $M^{\prime}$ respectively. Let $z$ be the class of $\beta_{1} \cdots \beta_{r}$. Since $U$ is nice this sequence already contains $A_{1} \cdots A_{R}$ and hence is persistent so $z \in R_{x}$. Let $z^{\prime}$ be the class of $\beta_{r+1} \cdots \beta_{N}$. By the same argument $z^{\prime}$ is persistent. In $M^{\prime}$ inside of, say, $[.5 n, .51 n]$ we find the block $A_{1} \cdots A_{R}$. By the universality property we can split this block into a segment in $L_{z}$ and another in $R_{z^{\prime}}$. Adding more 
to the left or right doesn't change the nature of this split. Thus there is an interval $\left[i_{r^{\prime}-1}^{\prime}, i_{r^{\prime}}^{\prime}\right)$ so that $\beta_{1}^{\prime} \cdots \beta_{r^{\prime}}^{\prime} \in L_{z}$ and $\beta_{r^{\prime}+1}^{\prime} \cdots \beta_{N^{\prime}}^{\prime} \in R_{z^{\prime}}$. Spoiler plays $i_{r^{\prime}}^{\prime}$ in response to $i_{r}$.

The class of $\beta_{1} \cdots \beta_{r}$ is $z$ and $z \in R_{x}$. The class $z^{\prime}$ of $\beta_{1}^{\prime} \cdots \beta_{r^{\prime}}^{\prime}$ is in $L_{z}$ and $R_{x}$. As $z \in L_{z} \cap R_{x}, z=z^{\prime}$. Thus $\left[1, i_{r}\right)$ under $M$ and $\left[1, i_{r^{\prime}}^{\prime}\right)$ under $M^{\prime}$ have the same Ehrenfeucht value. Thus Duplicator can respond successfully to the at most $t$ moves (including the initial move $m$ ) made in these intervals. Thus Spoiler may as well play the remaining $t-1$ moves on $M_{1}=<\left[i_{r}, n\right], \leq, U>$ and $M_{1}^{\prime}=<\left[i_{r^{\prime}}^{\prime}, n^{\prime}\right], \leq, U^{\prime}>$. These intervals have lengths $n_{1} \geq \frac{n}{3}$ and $n_{1}^{\prime} \geq \frac{n^{\prime}}{3}$ respectively. But now $M$ and $M^{\prime}$ are both nice with respect to $\delta_{1}=3 \delta$ - the sequence $A_{1} \cdots A_{R}$ still appears inside every interval of length $\delta n \leq \delta_{1} n_{1}$ in $M$ and $\delta_{1} n_{1}^{\prime}$ in $M^{\prime}$. Hence we can apply the same argument for the second move - for convenience still looking at Ehrenfeucht values with respect to the $t$ move game. After $t$ moves we still have nice $M_{t}, M_{t}^{\prime}$ with respect to $\delta_{t} \leq 10^{-2}$ so the arguments are still valid. But at the end of $t$ rounds Duplicator has won. 